\title{
Transection of the Common Carotid and Vertebral Arteries Following a Penetrating Neck Injury
}

\author{
Eun Ji Lee, Hojong Park * , Kyu Hyouck Kyoung, Sang Jun Park \\ Department of Surgery, Ulsan University Hospital, University of Ulsan College of Medicine, Ulsan, Korea
}

\section{Article history:}

Recevied: August 13, 2021

Revised: September 2, 2021

Accepted: September 3, 2021

\section{*Corresponding Author:}

Hojong Park

Department of Surgery, Ulsan University Hospital, University of Ulsan College of

Medicine 877 Bangeojinsunhwan-doro,

Dong-gu, Ulsan, Korea

Email: hjpark@uuh.ulsan.kr

ORCID

Eun ji Lee

https://orcid.org/0000-0001-8756-4287

Hojong Park

https://orcid.org/0000-0002-3293-9747

Kyu Hyouck Kyoung

https://orcid.org/0000-0002-2989-6520

Sang Jun Park

https://orcid.org/0000-0001-8112-6331

\begin{abstract}
A case of a 30-year-old man who was admitted following a penetrating neck injury is presented. A clinical examination and operative findings identified semi-comatose mentality, neck muscle laceration, and transection of the left common carotid and vertebral arteries and the internal jugular vein. During the operation, the carotid arteries were repaired by interposition grafting, and the internal jugular vein was ligated. The vertebral artery was managed by interventional embolization. Although the patient had some neurological deficit, he was discharged on postoperative Day 52 . This surgical case prompted a discussion regarding neurological outcomes, and surgical and endovascular treatment following vascular neck injury.
\end{abstract}

Keywords: carotid arteries, neck injuries, stroke

\section{Introduction}

Stab wounds to the neck are potentially lethal and often associated with vascular injury of the carotid artery and jugular vein. Simultaneous penetrating injuries to the carotid and vertebral arteries are uncommon, potentially causing severe bleeding and neurological deficits. We report a case of simultaneous carotid and vertebral artery transection managed by surgery and interventional angioembolization.

\section{Case Report}

A 30-year-old male with no history of illness presented to the Emergency Department after receiving a stab wound to the left neck area. Ambulance paramedics reported profuse bleeding from the neck when they arrived on the scene. The primary survey revealed a drunken, semi-comatose patient with normal sounds of breathing. Vital signs showed blood pressure which could not be measured, and tachycardia of 117 beats per minute on electrocardiogram monitoring. Initial volume resuscitation was started. A complete physical examination revealed an anteromedial stab wound to the left side of his neck with active bleeding. The internal, external, and common carotid arteries were completely transected, as was the internal jugular vein. The proximal and distal stumps of these vessels were clamped in the Emergency Room (Figure 1). Soon after, the systolic blood pressure was maintained above $90 \mathrm{mmHg}$. The patient was taken to the Operating Room where, under the exploration of the left side of the neck, the carotid arteries were repaired by interposition saphenous vein grafting, and the internal jugular vein was ligated. It took about 3 hours from neck injury to revascularization of carotid artery. However, there was arterial bleeding from a deeper portion, and the source 
was the transverse foramen of the cervical spine. Thus, the patient was taken to the intervention room for an angiogram, which showed discontinuation of the left mid-cervical vertebral artery (Figures $2 \mathrm{~A}$ and $2 \mathrm{~B}$ ). This area was the bleeding focus, and embolization was performed by an interventional radiologist (Figures 3A and 3B). After the vital signs stabilized, the neck muscles and wounds were repaired. On postoperative Day 2, the patient opened his eyes but could not make eye contact and did not obey instruction. On postoperative Day 3, a follow-up magnetic resonance imaging scan revealed extensive cortical acute infarct involving most of the anterior cerebral artery and middle cerebral artery territories with herniation but no hemorrhagic transformation (Figures 4 and 5). After performing the magnetic resonance imaging scan, tonic seizures occurred, and his eyeball deviated upward and to the left prompting a consultation with neurosurgery which resulted in the patient receiving coma therapy. On postoperative Day 10, the patient's eyes opened in response to pain stimulus, and right-sided weaknesses were observed. The patient was extubated on the same day but presented with global aphagia. Motor examination revealed Grade 2/5 strength in his right arm and $1 / 5$ in his right leg. Following rehabilitation, the patient was discharged on postoperative

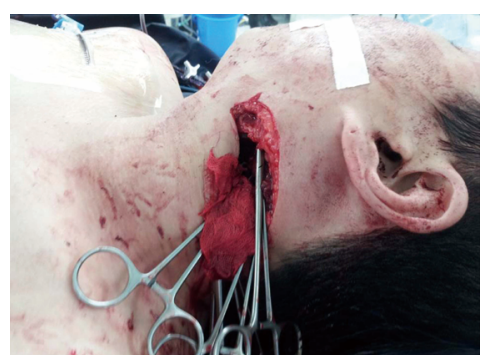

Figure 1. An anteromedial stab wound to the patient's left neck with active bleeding and complete Lt. internal and external carotid artery and internal jugular vein transection, vagus nerve transection, and sternocleidomastoid muscle and scalene muscle lacerations.

(A)

(B)

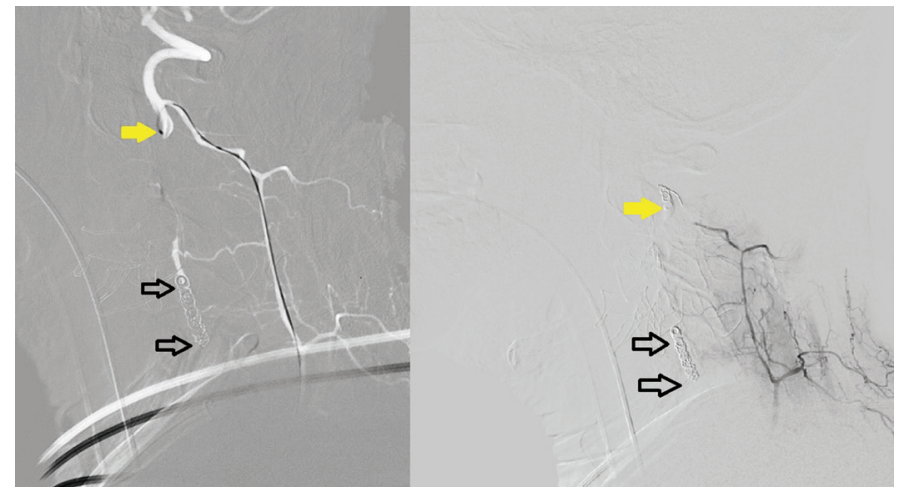

Figure 2. Conventional angiography of the left mid-cervical vertebral artery discontinuation (the bleeding focus); (A) anterior-posterior view; (B) lateral view.
(A)

(B)

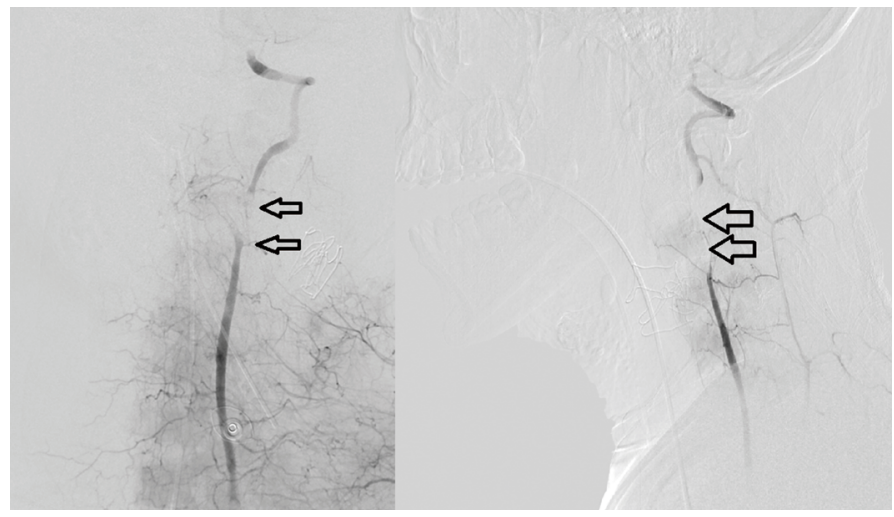

Figure 3. Artery embolization (A) proximal vertebral artery coil embolization (black arrow); (B) following distal vertebral artery embolization via the left thyrocervical trunk (yellow arrow).

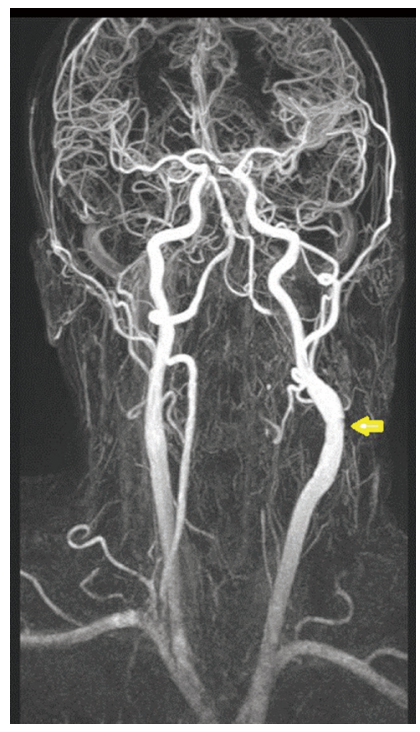

Figure 4. Postoperative magnetic resonance angiography showing patent left common to the internal carotid artery interposition graft (yellow arrow) and occlusion of the left vertebral artery.

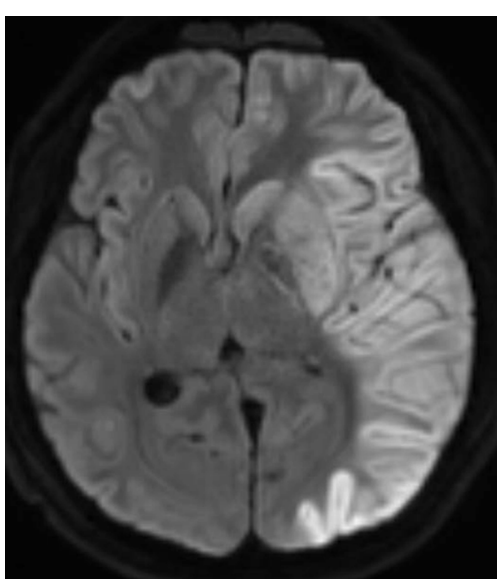

Figure 5. Postoperative diffusion-weighted magnetic resonance image showing extensive cortical acute infarct with herniation, multifocal small acute infarcts in the right frontal lobe, and no hemorrhagic transformation. 
Day 52. When the patient visited the Outpatient Department on postoperative Day 59, global aphagia was still observed, but motor examination revealed Grade 3/5 strength in his right arm and $4 / 5$ in his right leg.

\section{Discussion}

Carotid artery injuries are associated with high mortality (50\%) and persistent neurological deficits (80\%). Most patients with vascular neck injury died where the injury occurred or during transportation to the hospital. The usual pathology of neurological deficit following vascular neck injury is ischemic cerebral infarction caused by hypoperfusion or thromboembolism [1,2].

When examining a patient with injury to the vascular neck, it is important to check for "hard signs." Hard signs are reliable indicators for carotid artery injury and include an expanding or pulsatile hematoma, the absence of a pulse, the presence of bruit, and active external bleeding. Hemodynamically stable patients can be clinically observed and evaluated using radiological investigations [3]. Computed tomography angiography is the initial and the most informative diagnostic tool for patients with penetrating neck injuries who do not present with hard signs [4]. Noninvasive imaging such as magnetic resonance (MR) with angiography is also useful in the initial diagnosis and follow-up [5]. When carotid artery injury is suspected, and diagnostic procedures are impossible, then exploratory surgery is justified [1]. In this Case Report, the exploratory surgery was performed immediately without an evaluation using imaging because the patient's condition was unstable.

The mainstay of treatment should be early and aggressive resuscitation and revascularization. Patients undergoing revascularization generally benefit compared with patients who undergo ligation. The pathological basis is based on the principle of reperfusion of marginally perfused cerebral tissue. The recruitment of ischemic penumbra to normally functioning neurological tissue results in a better neurological outcome $[2,6]$. Thus, revascularization should be attempted whenever possible, regardless of the threat of hemorrhagic infarction, because hemorrhagic infarction rarely occurs and is unpredictable. Subsequent ischemic or hemorrhagic cerebral infarction are also unpredictable; the overall outcome of revascularizing the injured artery is superior to that achieved with ligation $[1,2,7]$.

The literature indicates that revascularization should be attempted in all patients, even those in a coma [8-10]. Immediate revascularization of coma patients appears to offer the best chance of improvement. A depressed mental status may be caused by shock, hypoxia, sedation, paralysis for airway control, or a combination of these [1,7]. The patient who is comatose and in profound shock should be treated like their neurological function will return to normal if the bleeding is controlled, blood volume restored, and the carotid artery injury promptly repaired [11]. Likewise, our patient had some improvement in neurological function after the critical period.

There are surgical or endovascular revascularization methods which are possible for vascular neck injury. However, surgical revascularization remains the gold standard for managing carotid artery transections, which can be performed by endto-end anastomosis, lateral sutures, and patch repair or bypass grafting (for larger injuries) [12,13]. Surgeons must consider during surgery that patients should be routinely draped for saphenous vein harvest. In patients with an incomplete circle of Willis, special attention should be paid to reduce ischemic infarction due to hypoperfusion during clamping and thromboembolism during surgical manipulation [2]. After achieving proximal and distal control, together with heparinization, visible thrombi should be carefully removed. Back-bleeding from the distal injured vessel indicates repair. Although shunting may decrease the ischemia duration, shunts may inherently increase thromboembolic events [2].

An extracranial internal carotid artery injury can be surgically challenging and occasionally requires ligation to prevent exsanguination. When ligation is performed, stroke and mortality rates are higher [1], likely related to the absence of collateral flow when the vessel is damaged or clamped, especially in patients with an incomplete circle of Willis and inaccessible bleeding in distal injuries. Variants in the circle of Willis anatomy are very common, with only $20 \%$ of patients (in anatomical studies) having a complete circle without any hypoplastic segments. In a trauma setting, having normal cerebral vascular anatomy is not enough to protect against a patient having a stroke [14]. Therefore, the indications for carotid artery ligation should be limited to the following circumstances: a very severe condition in the patient, a severe brain injury not associated with the carotid artery injury (e.g., a gunshot wound in the brain), a high Zone 3 internal carotid artery injury in the base of the skull when an anastomosis is not possible, and extensive distal-internal carotid artery thrombosis. In all other cases, the surgery should be restoration of carotid patency [1,7].

Endovascular therapy safely and effectively restores vascular luminal continuity or embolization if the surgical exposure is complicated $[4,5]$. The deep location of the vertebral artery within the neck makes injury relatively less common. However, this location also makes it difficult to detect and manage [15]. Additionally, a vertebral artery injury is surgically challenging because it is difficult to approach if a transected vertebral artery constricts and retracts into the foramen. In which case, angioembolization is more useful and effective. 
In conclusion, common carotid and vertebral artery transections are associated with high stroke and mortality rates. The mainstay of treatment should be immediate and aggressive resuscitation and revascularization. Revascularization should be attempted whenever possible. Endovascular treatment, including embolization, is safe and effective when the lesion is difficult to approach. When revascularization is too difficult, ligation of the injured carotid artery can be considered as a last lifesaving resort.

\section{Conflicts of Interest}

There are no conflicts of interest to declare.

\section{Acknowledgement}

We gratefully acknowledge the efforts of the following Ulsan University Hospital professors: Dongseok Yang MD, Eun Suk Park MD, Myung-Woul Han MD, Shang Hun Shin MD, Eun Mi Lee MD, Byung Ho Choi MD.

Their invaluable assistance made this work possible.

\section{Ethical Statement}

This research did not involve any human or animal experiment.

\section{References}

[1] Reva VA, Pronchenko AA, Samokhvalov IM. Operative management of penetrating carotid artery injuries. Eur J Vasc Endovasc Surg 2011;42(1):1620.

[2] du Toit DF, van Schalkwyk GD, Wadee SA, Warren BL. Neurologic outcome after penetrating extracranial arterial trauma. J Vasc Surg 2003;38(2):25762.

[3] Abu-Zidan EM, Abdel-Kader S, El Husseini R. Common carotid artery injury caused by a camel bite: Case report and systematic review of the literature. Ulus Travma Acil Cerrahi Derg 2014;20(1)0:59-62.

[4] Martinakis VG, Dalainas I, Katsikas VC, Xiromeritis K. Endovascular treatment of carotid injury. Eur Rev Med Pharmacol Sci 2013;17(5):673-88.

[5] Seth R, Obuchowski AM, and Zoarski GH. Endovascular repair of traumatic cervical internal carotid artery injuries: A safe and effective treatment option. AJNR Am J Neuroradiol 2013;34(6):1219-26.

[6] Liekweg WG Jr., Greenfield LJ. Management of penetrating carotid arterial injury. Ann Surg 1978;188(5):587-92.

[7] Ramadan F, Rutledge R, Oller D, Howell P, Baker C, Keagy B. Carotid artery trauma: A review of contemporary trauma center experiences. J Vasc Surg 1995;21(1):46-56.

[8] Brown MF, Graham JM, Feliciano DV, Mattox KL, Beall AC, DeBakey ME. Carotid artery injuries. Am J Surg 1982;144(6):748-53.

[9] Weaver FA, Yellin AE, Wagner WH, Brooks SH, Weaver AA, Milford MA. The role of arterial reconstruction in penetrating carotid injuries. Arch Surg 1988;123(9):1106-11.

[10] Richardson JD, Simpson C, Miller FB. Management of carotid artery trauma. Surgery 1988;104(4):673-80.

[11] Navsaria P, Omoshoro-Jones J, Nicol A. An analysis of 32 surgically managed penetrating carotid artery injuries. Eur J Vasc Endovasc Surg 2002;24(4):349-55.

[12] Thakore N, Abbas S, Vanniasingham P. Delayed rupture of common carotid artery following rugby tackle injury: A case report. World J Emerg Surg 2008;3:14.

[13] Gillespie DL. Comments regarding 'operative management of penetrating carotid artery injuries.' Eur J Vasc Endovasc Surg 2011;42(1):21.

[14] Shahan CP, Gray RI, Croce MA, Fabian TC. Impact of circle of Willis anatomy in traumatic blunt cerebrovascular injury-related stroke. Trauma Surg Acute Care Open 2017;2(1):e000086.

[15] de Castrol SMM, Christiaans SC, van den Berg R, Schep NWL. Minimal invasive management of traumatic transection of the vertebral artery. Springerplus 2014;3:206. 